University of Nebraska - Lincoln

DigitalCommons@University of Nebraska - Lincoln

2013

\title{
Evaluation of Sunlight-Exposed Pyrethroid-Treated Netting for the Control of Face Fly and Housefly (Diptera: Muscidae)
}

\author{
George W. Peck \\ Walter Reed Army Institute of Research, gwpeck5@gmail.com \\ Holly J. Ferguson \\ Washington State University \\ Jane T. LePage \\ Washington State University \\ Vincent R. Hebert \\ Washington State University \\ Sally D. O'Neal \\ Washington State University \\ See next page for additional authors
}

Follow this and additional works at: https://digitalcommons.unl.edu/usarmyresearch

Peck, George W.; Ferguson, Holly J.; LePage, Jane T.; Hebert, Vincent R.; O'Neal, Sally D.; and Walsh, Douglas B., "Evaluation of Sunlight-Exposed Pyrethroid-Treated Netting for the Control of Face Fly and Housefly (Diptera: Muscidae)" (2013). US Army Research. 259.

https://digitalcommons.unl.edu/usarmyresearch/259

This Article is brought to you for free and open access by the U.S. Department of Defense at DigitalCommons@University of Nebraska - Lincoln. It has been accepted for inclusion in US Army Research by an authorized administrator of DigitalCommons@University of Nebraska - Lincoln. 


\section{Authors}

George W. Peck, Holly J. Ferguson, Jane T. LePage, Vincent R. Hebert, Sally D. O'Neal, and Douglas B. Walsh 


\title{
Evaluation of sunlight-exposed pyrethroid-treated netting for the control of face fly and housefly (Diptera: Muscidae)
}

\author{
George W Peck, ${ }^{a, b *}$ Holly J Ferguson, ${ }^{a}$ Jane T LePage, ${ }^{c}$ Vincent R Hebert, ${ }^{c}$ \\ Sally D O'Neal ${ }^{\mathrm{a}}$ and Douglas B Walsh ${ }^{\mathrm{a}}$
}

\begin{abstract}
BACKGROUND: Face flies, Musca autumnalis De Geer (Diptera: Muscidae), and houseflies, Musca domestica L. (Diptera: Muscidae), have a significant impact on livestock and dairy production throughout North America. Pyrethroid insecticide efficacy can be affected by exposure to direct sunlight, and the rate of photodegradation is substrate and formulation dependent. Insecticidetreated netting (ITN) is finding new applications in crop and livestock production systems. A baseline study using long-duration no-choice assays has been carried out to gauge the effectiveness of ITN treated with $\beta$-cyfluthrin, $\lambda$-cyhalothrin and bifenthrin on face flies and houseflies.

RESULTS: After 12 weeks in direct sunlight, ITN treated with $\beta$-cyfluthrin was still highly insecticidal to face flies and houseflies, producing $100 \%$ mortality in petri dish assays. However, sunlight reduced the insecticidal activity of $\lambda$-cyhalothrin, with $3 \%$ of face flies and $\mathbf{5 0} \%$ of houseflies surviving after exposure to ITN that had been deployed for $\mathbf{1 0}$ weeks. Insecticidal activity was greatly reduced on bifenthrin-treated netting, with $\mathbf{2 0} \%$ of face flies and $\mathbf{5 0} \%$ of houseflies surviving in assays with netting deployed for only 3 weeks.
\end{abstract}

CONCLUSION: With careful choice of the pyrethroid applied, treated netting could be an important component of livestock integrated pest management programs focused on sustainable practices.

(c) 2013 Society of Chemical Industry

This article is a U.S. government work, and is not subject to copyright in the United States.

Keywords: pyrethroids; Musca autumnalis; Musca domestica; integrated pest management; dairy pest management

\section{INTRODUCTION}

Pest flies have a significant effect on livestock and dairy production throughout North America. ${ }^{1}$ Face flies, Musca autmnalis De Geer (Diptera: Muscidae), may impact on cattle behavior and productivity and can vector the causative agents of various cattle diseases such as pink eye, bovine thelaziasis, hemorrhagic bovine filariasis and brucellosis. ${ }^{2}$ Houseflies, Musca domestica L. (Diptera: Muscidae), can impact on cattle behavior by direct feeding on mucus secretions and may seriously affect the health and comfort of humans by vectoring enteric pathogens in dairy operations and adjacent habitations. ${ }^{1}$

Management of pest flies is difficult. For houseflies, chemical control methods include sticky traps, sugar- and pheromonebased insecticidal baits and adulticides formulated as knockdown or residual sprays. ${ }^{1}$ Control of adult houseflies with toxic baits has been an important tool in fly management for many years, ${ }^{3}$ mostly because of ease of use and long storage life. However, widespread and continuous use of chemical controls has led to worldwide insecticide resistance in many housefly populations. ${ }^{4}$ This resistance has been the focus of a large body of literature. ${ }^{5-9}$ For face flies, non-chemical management is focused on eliminating breeding sites through pasture harrowing to break up manure pats and increasing the stocking rate, whereby the animals break up the dung through their own trampling. In spite of the availability of many options in chemicals and formulations - dust bags, oilers, pour-ons, ear tags and feed-through insect growth regulators (IGRs) - logistical problems exist in getting enough active ingredient delivered to the pest. ${ }^{10}$

The insecticidal efficacy of pyrethroids can be reduced significantly upon exposure to sunlight and to a large degree depends on the pyrethroid's capability to absorb radiant energy and undergo photodecomposition. ${ }^{11}$ For example, $10-70$ times more photodecomposed resmethrin (compared with unexposed resmethrin) was needed to achieve similar $L_{50}$ values against houseflies. $^{12}$

\footnotetext{
Correspondence to: George W Peck, Walter Reed Army Institute, 530 Robert Grant Ave., Silver Spring, MD 20910, USA. E-mail: gwpeck5@gmail.com

a Environmental and Agricultural Entomology Laboratory, Irrigated Agriculture and Research Extension Center, Washington State University, Prosser, WA, USA

b Vector Control Department, Entomology Branch, Walter Reed Army Institute of Research, Silver Spring, MD, USA

c Food and Environmental Quality Laboratory, Washington State University Tri-Cities, Richland, WA, USA
} 
There is a large pyrethroid-treated ITN literature for biting flies, with recent papers focused on mosquitoes. ${ }^{13-15}$ Pyrethroidtreated netting has been evaluated for use in outdoor livestock containment facilities for control of tsetse flies (Glossina spp., Diptera: Glossinidae) ${ }^{16}$ and biting midges (Culicoides spp., Diptera: Ceratopogonidae) ${ }^{17}$ and as a peridomestic barrier for the control of phlebotomine sand flies (Diptera: Psychodidae). ${ }^{18}$ Although the authors could find no research on the use of ITN to control face flies, in a recent study in Ghana, the impact of deltamethrin-treated nets for the control of flies from the subfamilies Muscinae (but not face fly) and Stomoxyinae and the family Tabanidae attacking cattle in pens was evaluated. ${ }^{19}$

ITN is a novel approach to pest management in livestock operations and could be an important component of IPM strategic plans. Here, an evaluation of insecticidal activity of sun-exposed, pyrethroid-treated netting against face flies and houseflies is presented.

\section{MATERIALS AND METHODS}

\subsection{Insects}

Face flies were obtained from the Kansas State University laboratory colony (KSU), originally collected from northeastern Oklahoma in 1978, ${ }^{20}$ and from the University of Minnesota (MN 93) colony, which has been maintained in laboratory culture since 1993. Adult face flies were reared in Bug Dorm-1 insect rearing cages $(30 \times 30 \times 30 \mathrm{~cm}$, Product No. 1452; BioQuip Products, Rancho Dominguez, CA) with a 16:8 h L:D cycle, a temperature ranging from 20 to $25^{\circ} \mathrm{C}$ and a relative humidity $(\mathrm{RH})$ ranging from 45 to $55 \%$. Face fly larvae were reared on freshly thawed manure by standard methods. ${ }^{21}$ Adults were given water on paper wicks, table sugar and cow liver ad libitum. Oviposition was into freshly thawed cow manure ( $<6$ weeks old).

Houseflies were derived from the University of California at Riverside Entomology Department susceptible colony (UCR strain), which has been under laboratory culture since their collection in 1982 from a dairy in Mira Loma, California. Laboratory colonies were established, and larvae were reared on a standard medium ${ }^{22}$ containing $3 \mathrm{~L}$ of tap water, $4 \mathrm{~L}$ of wheat bran, $0.25 \mathrm{~L}$ of dry milk, $10 \mathrm{~mL}$ of nutritional yeast and $0.5 \mathrm{~L}$ of premoistened alfalfa pellets. Adults were reared using equal amounts of table sugar and powdered milk (separately presented) and tap water from paper wicks ad libitum. Adult houseflies were reared in Bug Dorm-1 insect rearing cages $(30 \times 30 \times 30 \mathrm{~cm}$, Product No. 1452; BioQuip Products, Rancho Dominguez, $C A$ ) in separate rearing rooms with a 16:8 h L:D cycle, a temperature ranging from 20 to $25^{\circ} \mathrm{C}$ and $\mathrm{RH}$ ranging from 45 to $55 \%$.

\subsection{ITN experimental design}

Bare Hand Vineyard and Crop bird netting made of 230 denier highdensity polyethylene (HDPE) monofilament (Nixalite of America, Inc., East Moline, IL) was used. This green netting is lightweight, durable, UV stable, rip resistant and easily installed in agricultural settings. It is presently sold for bird protection in vineyards, berry farms and row crops. It was chosen for use in the present ITN study because of its low cost and high durability.

Three pyrethroid insecticides were evaluated: $\beta$-cyfluthrin formulated as Tempo SC Ultra (118 $\mathrm{gL}^{-1}$; Bayer, Kansas City, MO), $\lambda$ cyhalothrin formulated as Grenade ER ( $97 \mathrm{~g} \mathrm{~L}^{-1}$; Intervet/ScheringPlough Animal Health, Roseland, NJ) and bifenthrin formulated as Capture 2 EC ( $251 \mathrm{~g} \mathrm{~L}^{-1}$; FMC, Philadelphia, PA). One $3 \times 5 \mathrm{~m}$ section of netting was soaked in the maximum-field-rate active ingredient $(\mathrm{Al})$ concentration of each of the formulations $(0.05 \%$ $\beta$-cyfluthrin, $0.06 \% \lambda$-cyhalothrin and bifenthrin). After soaking for 5 min, netting was air dried in a darkened room. Each piece was cut into two $1.5 \times 5 \mathrm{~m}$ sections for sun and shade treatments, packed separately in opaque plastic trash bags and transported to the deployment location.

ITNs were deployed near Prosser, Washington, at the Roza experimental farm (latitude $46^{\circ} 18^{\prime} \mathrm{N}$, longitude $119^{\circ} 44^{\prime} \mathrm{W}$ ). $\beta$-Cyfluthrin-treated netting was deployed on 12 June 2008. $\lambda$-Cyhalothrin-treated netting and bifenthrin-treated netting were deployed on 26 June 2008. The treated netting was allowed to hang vertically by draping it across stainless steel wire strung between $7 \mathrm{ft}(2.1 \mathrm{~m}) \mathrm{t}$-posts driven into the soil. Wires were strung $1.5 \mathrm{~m}$ (upper) and $0.2 \mathrm{~m}$ (lower) above the soil and were oriented east-west to maximize sunlight exposure. Netting was secured to protect against wind damage with plastic zip ties. Sunlight treatments were left exposed to full sun, while shade treatments (also oriented east-west) were placed under the roof of a plywood shed that had one wall on the south side. There were no other sources of shade.

A $50 \times 10 \mathrm{~cm}$ sample from each suspended section of pyrethroidtreated netting was collected and placed in a metallized pouch (Item No. 183-23; Associated Bag Co., Milwaukee, WI) at weekly intervals until the fourth week, and then at biweekly intervals until the 12th week after deployment. For the $\beta$-cyfluthrin-treated netting, additional samples were retrieved at 5 and 14 weeks after deployment. Immediately upon return to the laboratory, the pouches were sealed with a conventional clothes iron and frozen at $-80{ }^{\circ} \mathrm{C}$ until the day of biological assay on face flies ( $<6$ months in storage). After the netting needed for the bioassays had been removed, pouches were resealed and stored at $-80^{\circ} \mathrm{C}$ until transfer on ice to the WSU Food and Environmental Quality Laboratory (FEQL), where they were again stored at $-80{ }^{\circ} \mathrm{C}$ until netting samples were analyzed for insecticide concentration.

Total daily solar radiation $\left(\mathrm{MJ} \mathrm{m}^{-2}\right)$ and rainfall data were obtained from Washington State University's AgWeatherNet (AWN) (http://weather.wsu.edu/), using the station closest to the deployed ITN (WSU-Hamilton for first 30 days, Roza for days 31 to 90$)$.

\subsection{Assays with field-deployed netting}

For treated (positive) controls, dilutions (diluent was deionized water) of insecticides were mixed fresh each day for every bioassay. Treated control netting was dipped for $20 \mathrm{~s}$ in insecticide solution, shaken to remove excess insecticide and air dried on a flat surface for $1 \mathrm{~h}$ before beginning the assay. Negative controls consisted of netting dipped in deionized water only. All assays of fielddeployed netting were performed in $(9 \mathrm{~cm}$ diameter and $1 \mathrm{~cm}$ height) plastic petri dishes (VWR International, San Francisco, CA). Netting samples to fit $9 \mathrm{~cm}$ diameter petri dishes were taken from each archived sample of pyrethroid-treated netting. A cotton ball soaked in a $1 \%$ sucrose solution was placed in each dish. Each sampling date for each pyrethroid was replicated with four dishes (ten flies per dish), and all bioassays included four treated netting (positive control) dishes and four untreated netting (negative control) control dishes. All biological assays used 5-7-day-old flies of mixed sex, approximately 50:50 males:females, assuming that flies visiting ITN in the field would also be a mixture of males and females. Flies were aspirated out of rearing cages, anesthetized with $\mathrm{CO}_{2}$ and then placed into dishes. At $24 \mathrm{~h}$, flies unable to move (ataxic) even after dishes were tapped or lightly shaken were 
scored as dead. ${ }^{23}$ Laboratory temperatures ranged between 18 and $21{ }^{\circ} \mathrm{C}$, and $\mathrm{RH}$ ranged from 35 to $45 \%$ for the assays.

To save time and effort, netting samples from the latest dates for $\beta$-cyfluthrin shade treatment, $\lambda$-cyhalothrin sun and shade treatments and bifenthrin sun and shade treatments were assayed first. If the oldest and second oldest netting samples resulted in $100 \%$ mortality in the bioassays, samples from earlier dates were not tested against flies, assuming that earlier samples would also result in $100 \%$ fly mortality.

\subsection{Analysis of ITN insecticide concentrations}

Methods were developed by the WSU FEQL specifically for the evaluation of pyrethroid residues from the HDPE netting used in this study. ${ }^{24}$ The FEQL is a state-mandated food and environmental regulatory science facility that conducts studies under federal 40CFR Part 160 good laboratory practices (GLPs).

\subsubsection{ITN pyrethroid extraction}

When possible, duplicate samples were analyzed for each archived piece of pyrethroid-treated netting collected from the sections hung in sunny and shady conditions on each sample date. Two portions of netting were cut from each archived sample and weighed. The net samples were then placed in separate $25 \mathrm{~mL}$ Corex $^{\mathrm{TM}}$ tubes for extraction with $50 \%$ methylene chloride and $50 \%$ acetone. The samples were sonicated for $10 \mathrm{~min}$, and the extraction solvent was decanted into a round-bottom flask. The extraction by sonication was repeated a second time, and extracts were combined in the round-bottom flask for evaporation. The organic extract was evaporated to dryness by rotary evaporation. The samples were then reconstituted in $0.2 \%$ acetic acid in hexane and diluted appropriately for analysis. The acetic acid was necessary in the final solvent to minimize matrix (netting) enhancement effects.

\subsubsection{Analyte concentration determination}

$\beta$-Cyfluthrin, $\lambda$-cyhalothrin and bifenthrin concentrations were quantified using an Agilent $6890 \mathrm{~N}$ gas chromatograph (GC) (Agilent Technologies, Inc., Santa Clara, CA) with microelectron capture detection $(\mu \mathrm{ECD})$. A fused silica $30 \mathrm{~m} \times 0.32 \mathrm{~mm}$ I.D. $\times$ $0.25 \mu \mathrm{m}$ film thickness Agilent HP-5 chromatographic column was employed for compound separation and determination. Ultrapure helium was used as a carrier gas at a flow rate of $2.6 \mathrm{~mL} \mathrm{~min}{ }^{-1}$. Typical GC temperature conditions involved ramping the GC oven from 100 to $280^{\circ} \mathrm{C}$ at $20^{\circ} \mathrm{C} \mathrm{min}^{-1}$ and then holding for $5 \mathrm{~min}$. Compound identification was based on observed retention time from external calibration standards included in each analytical set. For each analytical set, at least one netting control (blank) and one recovery fortification sample were extracted in the same manner as the pyrethroid-impregnated netting samples. The recovery fortification sample was prepared by dripping a known volume of fortification solution for the compound of interest onto a portion of control netting.

The quantification of residual pyrethroid concentrations in the ITN was performed by electronic peak area measurement using Agilent Chemstation software (Agilent Technologies, Inc., Santa Clara, CA); values were compared with the linear regression from a minimum of four standards in the concentration range of sample extracts. To assure high quality during GC operation, all samples were bracketed with external calibration standards during the analytical set. For each analytical set, linearity and calibration standards were used to construct the calibration curve using a spreadsheet program (Microsoft Excel ${ }^{\circledR}$ ). The total residue concentration on the netting was calculated by multiplying the calculated concentration by the dilution factor and then dividing by the weight of the netting. The dilution factor is the equivalent of the ratio of final volume by the initial volume of sample extract. Efficiency of recovery ${ }^{24}$ (mean percentage validation recovery \pm 1 SD) was as follows: $\beta$-cyfluthrin $106 \pm 10 \%(n=12), \lambda$-cyhalothrin $106 \pm 10 \%(n=12)$ and bifenthrin $118 \pm 6 \%(n=12)$. While there were no samples from week 0 saved for residue analysis, data gathered during the FEQL preliminary storage stability study were used as proxy data for pyrethroid residue concentrations of netting drapes prior to exposure to field conditions. ${ }^{24}$ Three $0.3 \mathrm{~m}$ pieces of netting were soaked with the maximum label rate of each pyrethroid insecticide by a technique similar to that used in the field studies, and the initial mean residues for each pyrethroid were determined using methods described above.

\subsection{Assays to estimate lethal concentrations}

To serve as a baseline comparison with results from the field-aged ITN assays, lethal concentration ( $\mathrm{LC}_{50}$ ) assays were performed for the $\beta$-cyfluthrin, $\lambda$-cyhalothrin and bifenthrin formulations in the laboratory. Before soaking, netting was stretched across plastic petri dishes $(9 \mathrm{~cm}$ diameter and $1 \mathrm{~cm}$ height; VWR International, San Francisco, CA) and cut to fit. For $\mathrm{LC}_{50}$ assays, serial dilutions (diluent was deionized water) of insecticides were mixed fresh each day for every bioassay. Netting was soaked for $20 \mathrm{~s}$ in insecticide solution, shaken to remove excess insecticide and air dried on a flat surface for $1 \mathrm{~h}$ before beginning the assay. Negative controls consisted of netting dipped in deionized water only. Each dish received a cotton ball soaked in a $1 \%$ sucrose solution. Replication was at least three dishes (ten flies per dish) per dilution, and each insecticide was assayed on at least three different days. Concentration placement and sample size strongly influence the precision of $\mathrm{LC}_{50}$ estimates. ${ }^{25}$ The present assay design template used at least 320 flies and five or more concentrations per insecticide $\mathrm{LC}_{50}$ estimate. For $\beta$-cyfluthrin the concentrations ranged from 0.5 to $75.0 \mathrm{mg} \mathrm{kg}^{-1}$ (face fly) and from 5 to $125 \mathrm{mg} \mathrm{kg}^{-1}$ (housefly), for $\lambda$-cyhalothrin they ranged from 0.5 to $50.0 \mathrm{mg} \mathrm{kg}^{-1}$ (face fly) and from 2.5 to $100 \mathrm{mg} \mathrm{kg}^{-1}$ (housefly) and for bifenthrin they ranged from 0.5 to $50.0 \mathrm{mg} \mathrm{kg}^{-1}$ (face fly) and from 0.5 to $500 \mathrm{mg} \mathrm{kg}^{-1}$ (housefly).

\subsection{Statistical analysis}

Fly mortality data from assays on field-deployed netting were analyzed by analysis of variance using $R$ (The $R$ Project for Statistical Computing, v.2.13.1: http://www.r-project.org/). Insecticide residue data on field-deployed netting were subjected to stepwise multiple linear regression analysis using SAS (PROC REG). ${ }^{26}$ Dummy variables were utilized for sun exposure and pyrethroid factors. Transformations on fly percentage mortality data for field-deployed netting $(\arcsin \sqrt{ } p$, where $p$ is the proportion dead) and ITN residual pyrethroid concentration [ $\log _{10}$ $(x+1)]$ were performed before analysis. Tukey's HSD $(\alpha=0.05)$ was used to separate means after ANOVA. Mortality data from laboratory netting assays were analyzed using PoloPlus software. ${ }^{27}$ Control mortality was estimated from the dataset with the PoloPlus program. To test for differences among regression lines, likelihood ratio tests of equality and parallelism were performed in PoloPlus. Significant differences among LC $_{50}$ estimates of pyrethroid formulations were determined by non-overlap of $95 \%$ confidence limits. 

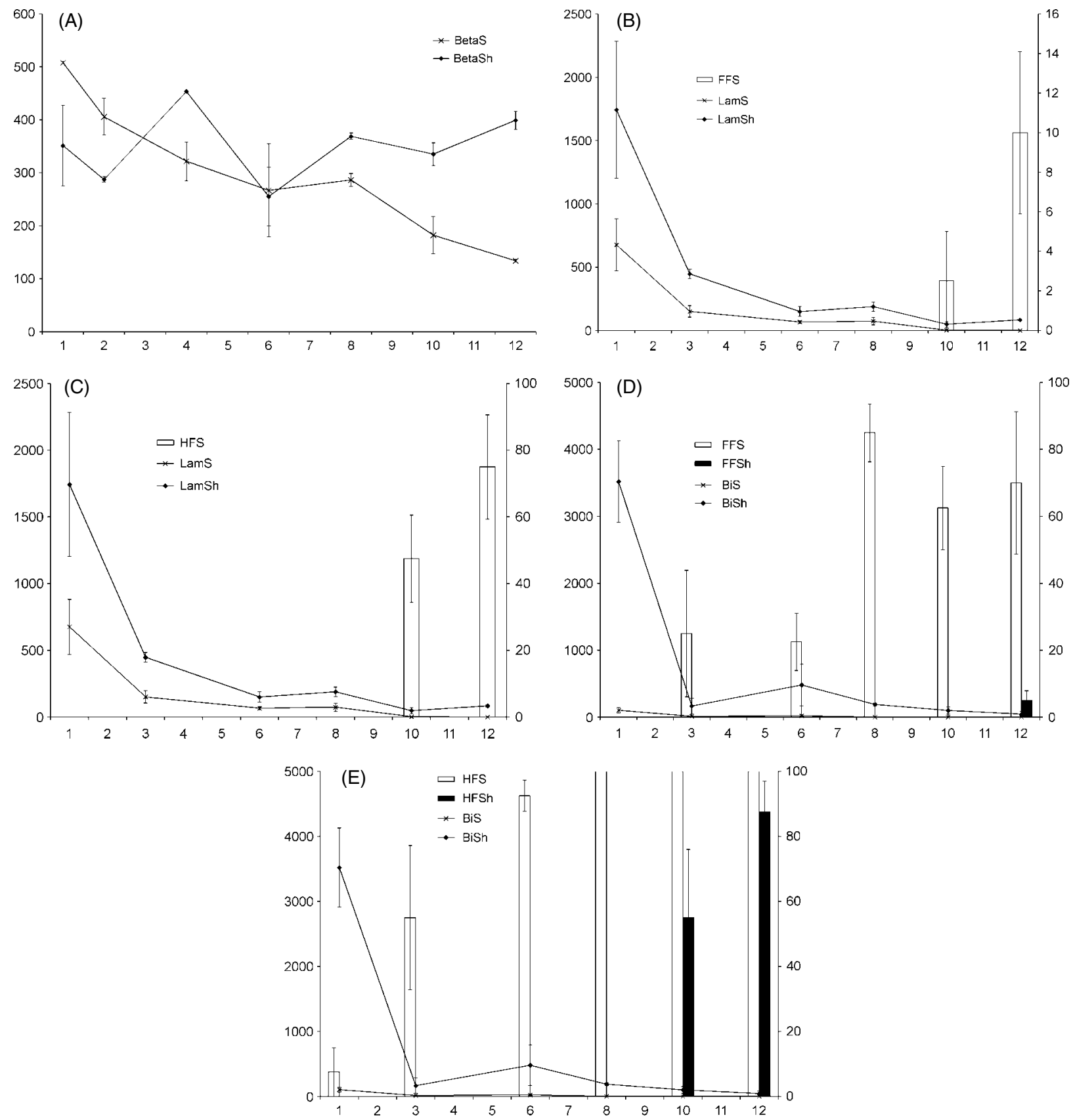

Figure 1. Pyrethroid residues (lines: sun/shade treatment) and percentage face fly and housefly survival (columns) versus time deployed for insecticidetreated netting hung in Prosser, Washington, June-September 2008. (A) $\beta$-cyfluthrin; (B) $\lambda$-cyhalothrin for face fly (FF); (C) $\lambda$-cyhalothrin for housefly (HF); (D) bifenthrin for face fly; (E) bifenthrin for housefly. $\mathrm{S}=$ sun treatment, $\mathrm{Sh}=$ shade treatment. Error bars are $\pm 1 \mathrm{SE}, n=2$ for residues, $n=4$ for survival assays.

\section{RESULTS}

\subsection{Field-deployed netting assays}

ITN efficacy against face flies and houseflies in plate assays was highly dependent on the number of weeks after deployment, sun exposure and pyrethroid formulation. Because of its stability under sunlight exposure, $\beta$-cyfluthrin-treated field-deployed netting (sun and shade) retained high insecticidal activity in petri dish assays ( $0 \%$ survival at $24 \mathrm{~h}$ ) against face flies and houseflies for all sampling dates and both treatments (Fig. 1A); the same was true for $\lambda$-cyhalothrin-treated netting from shade treatments (Fig. 1B). There was a strong 'weeks after deployment' effect on fly survival for sun-exposed netting treated with $\lambda$-cyhalothrin (face fly: $F_{7,48}=7.601, P<0.001$; housefly: $F_{7,48}=38.25, P<0.001$ ): netting deployed in sunlight for 10 weeks allowed $2.5 \pm 2.5 \%$ (mean $\pm \mathrm{SE} ; n=4$ plates) of face flies and $47.5 \%$ (SE $=13.1, n=4$ ) of houseflies to survive in $24 \mathrm{~h}$ plate assays, while the netting deployed in sunlight for 12 weeks allowed $10 \pm 4.1 \%$ of face flies and $75 \pm 15.6 \%$ of houseflies to survive in $24 \mathrm{~h}$ plate assays (Figs $1 \mathrm{~B}$ and $\mathrm{C})$.

For bifenthrin plate assays there was a strong 'sun exposure' effect (face fly: $F_{1,48}=65.99, P<0.001$; housefly: $F_{1,48}=133.7$, $P<0.001$ ), 'weeks after deployment' effect (face fly: $F_{7,48}=8.592$, $P<0.001$; housefly: $F_{7,48}=20.50, P<0.001$ ) and 'sun exposure $\times$ weeks after deployment' interaction (face fly: $F_{7,48}=6.931$, 


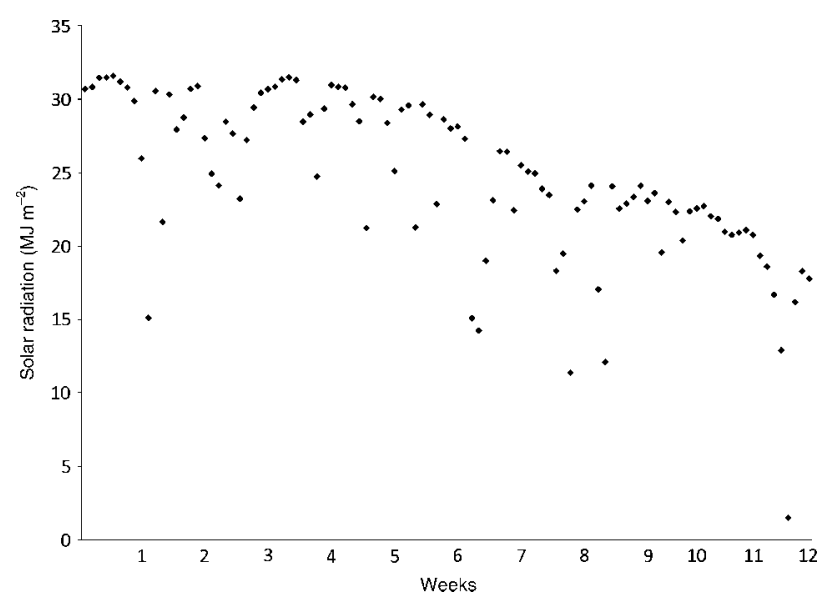

Figure 2. Total daily solar radiation $\left(\mathrm{MJ} \mathrm{m}^{-2}\right)$ over the 12 week deployment period, Prosser, Washington, 2008.

$P<0.001$; housefly: $\left.F_{7,48}=6.485, P<0.001\right)$. The strong sunlight effect on bifenthrin residual ITN concentrations and the rapid decrease in concentration observed after week 1 allowed large numbers of flies to survive plate assays (Figs 1D and E). For sunlight-exposed netting sampled on week 3 , plate assays on face flies gave $25.0 \pm 18.5 \%$ (mean $\pm \mathrm{SE} ; n=4$ plates) survival at $24 \mathrm{~h}$, and plate assays on houseflies gave $55.0 \pm 22.2 \%$ survival at $24 \mathrm{~h}$. By week 8 , the decrease in bifenthrin ITN residual concentration allowed $85.0 \pm 8.7 \%$ of assayed face flies to survive and $100.0 \pm$ $0.0 \%$ of houseflies to survive, a pattern that continued until the termination of sampling at week 12 . Shade-exposed bifenthrintreated netting retained its effectiveness ( $0 \%$ surviving) in assays through week 8. However, week 10 plate assays on houseflies showed $55.0 \pm 21.0 \%$ (mean $\pm \mathrm{SE} ; n=4$ plates) survival at $24 \mathrm{~h}$. Similar but smaller effects were seen in week 12 plate assays on face flies: $5.0 \pm 2.9 \%$ survival at $24 \mathrm{~h}$.

\subsection{ITN residual pyrethroid analysis}

Over the course of the experiment, total daily solar radiation ranged between 1.5 and $31.6 \mathrm{MJ} \mathrm{m}^{-2}$ day $^{-1}$, following the typical seasonal pattern (Fig. 2). Approximately 15 cloudy days occurred during the 12 week deployment, with total rainfall measured at $13.0 \mathrm{~mm}$.

Data gathered during the FEQL preliminary storage stability study served as proxy data for pyrethroid residue concentrations of netting drapes prior to exposure to field conditions (week 0). ${ }^{24}$ Initial mean ( $\pm \mathrm{SE}$ ) residues for each pyrethroid were (in $\mathrm{mg} \mathrm{kg}^{-1}$ ): $\beta$-cyfluthrin, $223.0 \pm 46.0 ; \lambda$-cyhalothrin, $990.2 \pm 70.1$; bifenthrin, $5087 \pm 797$. The initial concentration of bifenthrin was significantly greater than the $\beta$-cyfluthrin and $\lambda$-cyhalothrin initial values in the storage stability study $(F=31.95, \mathrm{df}=2, P=0.001)$.

Based on stepwise multiple linear regression analysis, insecticide residue concentration was dependent on number of weeks after deployment, sun exposure and pyrethroid formulation (Table 1). Pyrethroid treatments were different with respect to their intercepts, but not to their slopes. From 1 to 14 weeks after deployment, the residual concentrations in the $\beta$-cyfluthrintreated netting exposed to the sun decreased by $64 \%$ (from 507 . 95 to $183.25 \mathrm{mg} \mathrm{kg}^{-1}$ ) (Fig. $1 \mathrm{~A}$ ); during a similar period (1 to 12 weeks after deployment), the concentrations of sun-exposed $\lambda$-cyhalothrin and bifenthrin residues decreased by 100 and $96 \%$ respectively, for $\lambda$-cyhalothrin from 676.05 to $0.80 \mathrm{mg} \mathrm{kg}^{-1}$ (Figs 1B
Table 1. Results of multiple linear regression analysis in insecticide residues to determine effects of weeks after deployment, sun versus shade treatment and pyrethroid ( $\beta$-cyfluthrin, bifenthrin and $\lambda$-cyhalothrin) on insecticide-treated HDPE netting hung in field conditions near Prosser, Washington, June to September 2008

\begin{tabular}{|lrccc|} 
Variable & Estimate & Standard error & \multicolumn{1}{c}{$F$} & $P$ \\
\hline Intercept & 3.40 & 0.13 & $679.22<0.0001$ \\
Weeks after & -0.09 & 0.01 & $50.45<0.0001$ \\
$\quad$ deployment (wad) & & & & \\
Sun versus shade $(s)$ & -0.66 & 0.11 & $40.04<0.0001$ \\
Pyrethroid ( $p$ 1) & -0.49 & 0.13 & 15.06 & 0.0002 \\
Pyrethroid ( $p 2)$ & -0.78 & 0.13 & $37.62<0.0001$ \\
\hline
\end{tabular}

Residue values were $\log _{10}(x+1)$ transformed before analysis. Equation: $\hat{y}=3.40-0.09$ wad $-0.66 s-0.49 p 1-0.78 p 2+\varepsilon$, where wad $=$ weeks after deployment; $s=$ sun versus shade treatment; $p 1$ and $p 2=$ dummy variables for pyrethroid formulation. Residual mean square error $=0.22552$.

and C) and for bifenthrin from 104.0 to $4.0 \mathrm{mg} \mathrm{kg}^{-1}$ (Figs 1D and E). This decrease on netting exposed to the sun was most rapid when solar irradiation was strongest (week 1 to week 6), with more moderate decrease in concentration after 6 weeks. In contrast, the concentration of $\beta$-cyfluthrin residue on netting in shade remained fairly constant over time, with only a $25 \%$ decrease from 1 to 14 weeks after deployment, from 351.5 to $262.4 \mathrm{mg} \mathrm{kg}^{-1}$ (Fig. 1A). The residue concentration of $\lambda$-cyhalothrin-and bifenthrin-treated netting in the shade dropped by 95 and $99 \%$ from week 1 to 12 respectively, $\lambda$-cyhalothrin from 1743.8 to $83.8 \mathrm{mg} \mathrm{kg}^{-1}$ (Figs 1B and C) and bifenthrin from 3521.6 to $47.9 \mathrm{mg} \mathrm{kg}^{-1}$ (Figs 1D and E).

\subsection{Lethal concentration estimates}

Within the series of assays on freshly soaked netting there was a great variation in LC estimates between species and within species for insecticides (Table 2). More than 3.9 times as much $\beta$-cyfluthrin, 2.8 times as much $\lambda$-cyhalothrin and 216.2 times as much bifenthrin were required to attain the same level of $L C_{50}$ for houseflies as for face flies.

Face fly probit models generated for the three pyrethroid formulations differed significantly with respect to their slopes and intercepts $\left(\chi^{2}=124.0, \mathrm{df}=4, P<0.05\right)$. Based on the overlapping of confidence intervals, the face fly $\mathrm{LC}_{50}$ values of $\beta$-cyfluthrin and $\lambda$-cyhalothrin were not different from each other, but the $\mathrm{LC}_{50}$ of bifenthrin was significantly lower than the $\mathrm{LC}_{50}$ for the other two pyrethroids (Table 2). Housefly probit models generated for the three pyrethroid formulations differed significantly with respect to their slopes and intercepts $\left(\chi^{2}=459.0, \mathrm{df}=4, P<0.05\right)$. Based on the non-overlapping of $95 \%$ confidence intervals, the housefly $\mathrm{LC}_{50}$ values of $\beta$-cyfluthrin, $\lambda$-cyhalothrin and bifenthrin were significantly different from each other.

\section{DISCUSSION}

To the present authors' knowledge, this is the first bioassay study with face flies and houseflies subjected to sunlight-exposed netting dipped in pyrethroids to be coupled with analysis of temporal decline of residue on netting. It is also the first evaluation of pyrethroid-dipped netting against face flies and houseflies. The present preliminary study shows that sunlight-induced decay of residue on netting and, concomitantly, ITN efficacy in petri dish assays against these flies depend greatly upon the type of 
Table 2. Results of probit analysis to estimate the insecticidal activity of netting soaked in one of three pyrethroid active ingredients ( $\beta$-cyfluthrin, $\lambda$-cyhalothrin and bifenthrin) against face flies and houseflies

\begin{tabular}{|c|c|c|c|c|c|c|c|}
\hline Species & Strain & Insecticide & Conc. range $\left(\mathrm{mg} \mathrm{kg}^{-1}\right)$ & $n$ & Slope (SE) & $\mathrm{LC}_{50}\left(\mathrm{mg} \mathrm{kg}^{-1}\right)(95 \% \mathrm{CL})$ & $\chi^{2}(\mathrm{df})$ \\
\hline \multirow[t]{3}{*}{ Face fly } & Kansas & $\beta$-Cyfluthrin & $0.5-75$ & 869 & $2.25(0.14)$ & $8.8(4.4-14.4)$ & $24.28(4)$ \\
\hline & Kansas & $\lambda$-Cyhalothrin & $0.5-50$ & 320 & $2.27(0.29)$ & $6.5(4.9-8.2)$ & $2.15(3)$ \\
\hline & $\mathrm{MN} 3^{\mathrm{a}}$ & Bifenthrin & $0.5-50$ & 690 & $0.85(0.11)$ & $1.0(0.1-2.2)$ & $56.33(9)$ \\
\hline \multirow[t]{3}{*}{ Housefly } & UCR & $\beta$-Cyfluthrin & $5.0-125$ & 1240 & $2.30(0.115)$ & $34.4(29.02-40.53)$ & $7.65(4)$ \\
\hline & UCR & $\lambda$-Cyhalothrin & $2.5-100$ & 1320 & $2.14(0.105)$ & $17.9(12.93-24.58)$ & $41.19(6)$ \\
\hline & UCR & Bifenthrin & $0.5-500$ & 1620 & $1.69(0.102)$ & $216.2(161.71-276.43)$ & $24.12(8)$ \\
\hline
\end{tabular}

pyrethroid formulation applied to the netting. Previous evaluation of pyrethroid insecticides confirms the effectiveness of pyrethroids, especially when they are carefully rotated with other classes of insecticides to avoid resistance build-up. ${ }^{28,29}$ Although there is no recent pyrethroid research on face flies, these compounds are still recommended for fly control in livestock production and dairy systems. ${ }^{30}$

The lethal effect for face flies and houseflies to sun-exposed $\beta$-cyfluthrin-treated netting for the duration of the 12 week deployment period suggests that using pyrethroid-treated netting with this formulation has potential as a management tool for field populations of these flies. Similarly, the low survivorship for face flies to sun-exposed $\lambda$-cyhalothrin-treated netting indicates that this formulation has management tool potential as well. Differences in $\mathrm{LC}_{50}$ across pyrethroid treatments for face flies should be viewed with caution, as the MN93 strain was substituted for bifenthrin assays. The tenfold difference in bifenthrin $\mathrm{LC}_{50}$ assays may be attributed to differences in resistance between strains. Differences in $\mathrm{LC}_{50}$ across pyrethroid treatments for houseflies showed a fourfold difference between bifenthrin and the two other pyrethroids. The high variability seen in the residue data within and between sample dates was probably because of the cost-saving decision to restrict the number of samples tested from each treatment. Nonetheless, the differences in pyrethoid stability after exposure to sunlight suggests that the persistence and insecticidal activity of the netting will depend greatly on insecticide formulation. It is not known why bifenthrin was far less persistent on the netting than the other two pyrethroids. It is speculated that bifenthrin degrades faster than the other two pyrethroids in direct sunlight, and that certain unique properties of the bifenthrin formulation reduced its ability to adhere to the plastic (HDPE) netting. Conversely, certain additives in the $\beta$-cyfluthrin formulation may have imparted better adhesion to the plastic netting matrix. Additional insecticide formulations, including those with neonicotinoids ${ }^{3}$ and semicarbazones ${ }^{31}$ as active ingredients, should be tested on this netting matrix.

The dynamics of fly interaction with netting is an important factor for determining the dose of insecticide delivered. In the present study, flies tended to move around within the petri dish, periodically walking over and on the netting. No differences in fly behavior (compared with control assays) such as repellency or irritancy ${ }^{32}$ were observed within the present no-choice testing arenas for any of the tested pyrethroid formulations. This study scored survival for each test after constant exposure to ITN at $24 \mathrm{~h}$, an exposure time that may overestimate the dose acquired by flies interacting with ITN in field conditions during equivalent time intervals.
As with mosquitoes, housefly resistance to pyrethroid insecticides is probably the most important long-term threat to the effectiveness of ITNs. This resistance is mediated mostly by a reduced sodium channel sensitivity to pyrethroids, resulting from one or more point mutations in sodium channel proteins. ${ }^{33}$ These pyrethroid resistance mutations may or may not incur a fitness $\operatorname{cost}^{34}$ and have arisen de novo in housefly populations worldwide. ${ }^{35}$ Because of resistance, future ITN studies should include non-pyrethroid housefly insecticides. While there is a large literature for insecticide resistance in $M$. domestica, no published evidence indicating similar development of pyrethroid resistance in face flies was found. As with any residual insecticide dispensing system, insecticide resistance can be avoided or slowed down by limiting exposure time and developing rotation systems among classes of insecticides applied to the netting, similarly to what is now recommended with insecticide-impregnated ear tags. ${ }^{36}$

Because it is generally agreed that fly IPM is the integration of chemical, biological and cultural control methods, it is suggested that ITN might be an important addition to the suite of fly IPM tools currently available. The results of the small-scale experiments reported here have established the ability of HDPE monofilament soaked in label concentrations of $\beta$-cyfluthrin and $\lambda$-cyhalothrin formulations to be insecticidal to face flies, even after 3 months in direct sunlight. Future studies should involve large-scale field trials as well as studies designed to examine the effects of different concentrations and different fly exposure periods to ITN in assays. Optimally, the netting may be most effective when deployed in areas where flies are known to rest away from their hosts, such as around the perimeter of corrals and pasture fences, and over shrubbery and trees. Perhaps ITN could be utilized as walk-through screening for entrances into run-in sheds or other shelters. While both pyrethroids could be used in all of the aforementioned areas, $\beta$-cyfluthrin has an advantage over $\lambda$-cyhalothrin, with no label restriction on use in milk rooms. Organic producers may benefit as well if the ITN can be deployed with approved chemistries or as a perimeter barrier beyond the buffer limit imposed by regulations.

Sustainable livestock and dairy production systems should be economically viable, environmentally sound and socially responsible. While resistance continues to be an issue, ${ }^{7,8}$ ITN has been used with great success in livestock systems, ${ }^{16-19}$ and careful choice of insecticide formulation, rotation among insecticide classes and minimization of the ITN deployment interval are sure to be central goals of a comprehensive resistance management program. Adoption of ITN technology, with its low cost and durability, precise application method and widening societal acceptance, may be one step towards sustainable livestock production systems. 


\section{ACKNOWLEDGEMENTS}

The authors thank the staff of the Environmental and Agricultural Entomology Laboratory for their technical assistance. This work was funded by a USDA Risk Management Agency (RMA) grant and a USDA Pest Management Alternatives Program (PMAP) grant to DB Walsh.

\section{REFERENCES}

1 Moon RD, Muscid flies, in Medical and Veterinary Entomology, 1st edition, ed. by Mullen G and Durden L. Academic Press, San Diego, CA, pp. 279-301 (2002).

2 Krafsur ES and Moon RD, Bionomics of the face fly, Musca autumnalis. Annu Rev Entomol 42:503-523 (1997).

3 Butler S, Gerry A and Mullens B, House fly (Diptera: Muscidae) activity near baits containing (Z)-9-tricosene and efficacy of commercial toxic fly baits on a southern California dairy. J Econ Entomol 100:1489-1495 (2004).

4 Keiding J, Review of the global status and recent development of insecticide resistance in field populations of the housefly Musca domestica (Diptera: Muscidae). Bull Entomol Res 89:S7-S67 (1999).

5 Kaufman P, Scott J and Rutz D, Monitoring insecticide resistance in house flies (Diptera: Muscidae) from New York dairies. Pest Manag Sci 57:514-521(2001).

6 Scott J, Alefantis T, Kaufman P and Rutz D, Insecticide resistance in house flies from caged-layer poultry facilities. Pest Manag Sci 56:147-153 (2000).

7 Khan H, Akram W and Shad S, Resistance to conventional insecticides in Pakistani populations of Musca domestica L. (Diptera: Muscidae): a potential ectoparasite of dairy animals. Ecotoxicology, 22:522-527 (2013).

8 Akiner $M$ and Caglar S, Monitoring of five different insecticide resistance status in Turkish house fly Musca domestica L. (Diptera: Muscidae) populations and the relationship between resistance and insecticide usage profile. Turkiye Parazitol Derg 36(2):87-91 (2012).

9 Darbro J and Mullens B, Assessing insecticide resistance and aversion to methomyl-treated toxic baits in Musca domestica L. (Diptera: Muscidae) populations in southern California. Pest Manag Sci 60:901-908 (2004).

10 Campbell JB, Face fly control guide. Extension NebGuide G1204, University of Nebraska, Lincoln, NE (2006).

11 Spurlock F and Lee M, Synthetic pyrethroid use patterns, properties, and environmental effects. ACS Symposium Series, Environmental Monitoring Branch, California Department of Pesticide Regulation, Sacramento, CA, pp. 3-25 (2008).

12 Ueda K, Gaughan LC and Casida JE, Photodecomposition of resmethrin and related pyrethroids. J Agric Food Chem 22:212-220 (1974).

13 Erlanger TE, Enayati AA, Hemingway J, Mshinda H, Tami A and Lengeler $C$, Field issues related to effectiveness of insecticide-treated nets in Tanzania. Med Vet Entomol 18:153-160 (2004).

14 Msangi S, Lyatuu E, Masenga C and Kihumo E, The effects of washing and duration of use of long-lasting insecticidal nets (PermaNets) on insecticidal effectiveness. Acta Tropica 107:43-47 (2008).

15 Sahu S, Vijayakumar T, Kalyanasundaram M, Subramanian S and Jambulingam $\mathrm{P}$, Impact of lambdacyhalothrin capsule suspension treated bed nets on malaria in tribal villages of Malkangiri district, Orissa, India. Indian J Med Res 128:262-270 (2008).

16 Bauer B, Gitau D, Oloo FP and Karanja SM, Evaluation of a preliminary title to protect zero-grazed dairy cattle with insecticide-treated mosquito netting in Western Kenya. Trop Anim Hlth Prod 38:29-34 (2006).
17 Calvete C, Estrada R, Miranda M, Del Rio R, Borras D, Beldron F et al., Protection of livestock against bluetongue virus vector Culicoides imicola using insecticide-treated netting in open areas. Med Vet Entomol 24:169-175 (2010).

18 Faiman R, Cuno R and Warburg A, Control of phlebotomine sand flies with vertical fine-mesh nets. J Med Entomol 46:820-831(2009).

19 Maia M, Clausen P-H, Mehlitz D, Garms R and Bauer B, Protection of confined cattle against biting and nuisance flies (Muscidae: Diptera) with insecticide-treated nets in the Ghanaian forest zone at Kumasi. Parasitol Res 106:1307-1313 (2010).

20 Broce $A$ and Elzinga R, Comparison of prestomal teeth in the face fly (Musca autumnalis) and the house fly (Musca domestica) (Diptera: Muscidae). J Med Entomol 21:82-85 (1984).

21 Moon R, Effects of larval competition on face fly. Environ Entomol 9:325-330 (1980).

22 Mandeville J, Mullens B and Meyer J, Rearing and host age suitability of Fannia canicularis (L.) (Diptera: Muscidae) for parasitization by Muscidifurax zaraptor Kogan and Legner (Hymenoptera: Pteromalidae). Can Entomol 120:153-159 (1988).

23 Kaufman P, Gerry A, Rutz D and Scott J, Monitoring susceptibility of house flies (Diptera: Muscidae) in the United States to imidacloprid. J Agric Urban Entomol 23:195-200 (2006).

24 LePage J, Evaluation of pyrethroid pesticides from field-aged pesticide impregnated netting. Analytical summary report, FEQL Study No. 0509, Washington State University Food and Environmental Quality Laboratory, Richland, WA (2009).

25 Robertson J, Smith K, Savin N and Lavigne R, Effects of dose selection and sample size on the precision of lethal dose estimates in dose-mortality regression. J Econ Entomol 77:833-837 (1984).

26 Littell R, Stroup W and Freund R, SAS ${ }^{\circledR}$ for Linear Models, 4th edition. SAS Institute, Cary, NC (2002).

27 Robertson J, Preisler H and Russell R, PoloPlus User's Guide, Version 2.0. LeOra Software, Petaluma, CA (2007).

28 Kaufman P, Scott J and Rutz D, Monitoring insecticide resistance in house flies (Diptera: Muscidae) from New York dairies. Pest Manag Sci 57:514-521 (2001).

29 Scott J, Alefantis T, Kaufman P and Rutz D, Insecticide resistance in house flies from caged-layer poultry facilities. Pest Manag Sci 56:147-153 (2000).

30 Gamroth M and Pirelli G, Fly control, in Pacific Northwest Insect Management Handbook, ed. by Hollingsworth C. University of Oregon, Extension and Experiment Station Communications, Corvallis, OR (2010).

31 Mullens B and Gerry A, Field and laboratory trials of a novel metaflumizone house fly (Diptera: Muscidae) bait in California. J Econ Entomol 103(2):550-556 (2010).

32 Roberts D, Alecrim W, Hshieh P, Grieco J, Bangs M, Andre R, et al, A probability model of vector behavior: effects of DDT repellency, irritancy, and toxicity in malaria control. $J$ Vector Ecol 25:48-61 (2000).

33 Dong K, Insect sodium channels and insecticide resistance. Invert Neurosci 7(1):17-30 (2007).

34 Rinkevich F, Leichter C, Lazo T, Hardstone M and Scott J, Variable fitness costs for pyrethroid resistance alleles in the house fly, Musca domestica, in the absence of insecticide pressure. Pestic Biochem Physiol, http://dx.doi.org/10.1016/j.pestbp.2013.01.006 (2013).

35 Rinkevich F, Hedtke S, Leichter C, Harris S, Su C and Brady S, Multiple origins of $k d r$-type resistance in the house fly, Musca domestica. PLoS One 7(12):28 (2012).

36 Schreder $\mathrm{P}$ and Pirelli G, Pacific Northwest Insect Management Handbook. Beef Cattle Pests. [Online]. Available: http://uspest.org/ pnw/insects (2010). 